These seeds were not studied at the time, but they are now seen to agree excellently with those of Ranunculus. They are, properly speaking, achenes, about $\mathrm{I} .7 \mathrm{~mm}$. long and I $\mathrm{mm}$. broad, with a fairly long moderately curved beak. The general form approaches that of $R$. pennsylvanicus, but the achene is less robust. The fossil may be known as $R$. florissantensis n. sp. Ranunculus has a single seed in the achene. It has been definitely determined for $R$. acfis at least that there is only one ovule. Our fossils, however, very distinctly show two, after the manner of Hydrastis. They were evidently small and dry at maturity, as in Ranunculus, and the most mature ones contain only one seed, dark and clearly outlined. It would be worth while to investigate the immature achenes of numerous species of Ranunculus to determine whether any start with two ovules, one aborting; as I have determined to be the case in Malvastrum. Heer has described a very similar Ranunculus seed (achene) from the Miocene of Oeningen in Baden.

T. D. A. Cockerell.

University of Colorado, Boulder, Colorado, December 8.

\section{The Absorption of Fluorescing Sodium Vapour.}

AcCORDING to Bohr's theory concerning the origin of spectral lines, electrons in the atoms of sodium vapour under the influence of exciting $D$ radiation are displaced from their normal $1 \cdot 5 \mathrm{~S}$ orbit to the $2 p$ orbits. During the return of the electrons to their normal orbit the so-called "resonance" radiation, first observed by Wood (Phil. Mag., November, I905, and "Researches in Physical Optics," part 2, p. I66), is re-emitted as fluorescent light. It has been suggested, therefore; by more than one observer (Foote and Meggers, Phys, Rev., vol. I5, part 4, p. 323, and Phil. Mag., vol. 40, p. 80, 1920) that fluorescing sodium vapour should absorb lines of the first and second subordinate series $2 p_{12}-m s$ and $2 p_{12}-m d$. To test this point I have

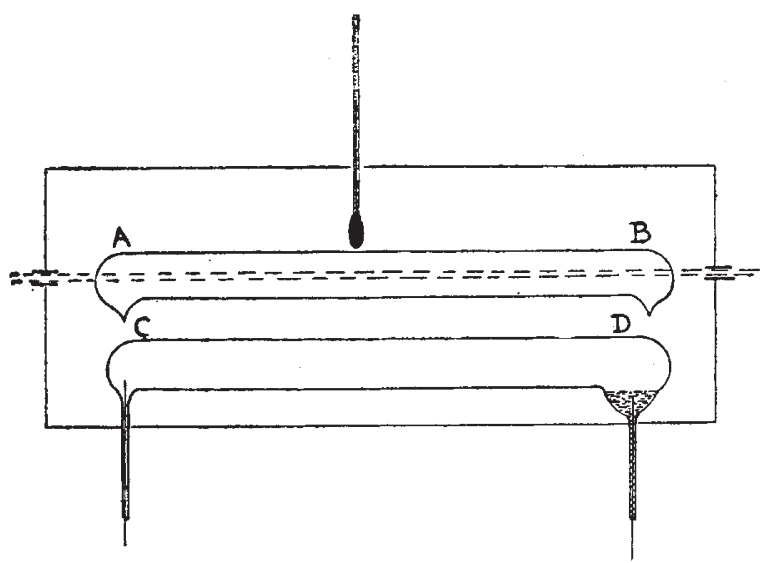

FIG. x.

made use of the experimental arrangement outlined below. While a negative result was obtained, it seems worth while to record the trial, along with one or two suggestions for a more rigid test which I am not able to make at present.

A narrow beam of light from a Nernst lamp traversed the tube $A B$, some $25 \mathrm{~cm}$. long, into which some sodium had been distilled in high vacuum (Fig. I). By means of a lens the light was focussed on the slit of a Hilger constant-deviation spectroscope. Directly below the sodium tube was a sodium-potassium alloy "lamp" $\mathrm{CD}$, a slight modification of the type reNO. 2724 , VOL. IOg] centıy described by Neuman (Proc. Phys. Soc. London, vol. 33, part 2, February I5 $_{5}$ 192 I). Both tubes were enclosed in an electric oven, by means of which they could easily be heated to $300^{\circ} \mathrm{C}$. or higher. For two reasons it seemed to the writer that this lamp should be most suitable. In the first place, resonance can best be excited by very narrow spectral sources, and it had been shown that the light from the lamp consisted almost entirely of $D$ lines of narrow width. Again, it could be operated at temperatures at which resonance is obtained in sodium vapour, so that it was possible to place the two tubes side by side in the same oven.

At temperatures ranging from $200^{\circ} \mathrm{C}$. to $300^{\circ} \mathrm{C}$., therefore, observations were made to see if there was any difference in the absorption spectrum when the exciting lamp was "on" and "off." The D absorption lines were easily visible, but not the slightest difference could be detected in the two cases. It is possible, however, that with an improved arrangement the absorption looked for might occur. The lines of the subordinate series most likely to be absorbed are the first members, which, however, are in the infra-red region and could not be observed visually. A much better test, therefore, would consist in photographing with dicyanine-stained plates in the hope of observing absorption of the doublet $\lambda 8195$ and $\lambda 8 \mathrm{I} 84$. Again, the intensity of the exciting light may not have been great enough to put a sufficiently large number of atoms in the desired state. This difficulty would be lessened by the use of two or three lamps, or possibly by adapting for use with sodium some such arrangement as was used by Füchtbauer (Phys. Zeit., vol. 21, November I and is, I920) for observing resonance in mercury vapour. Finally, a longer absorption tube obviously would be more desirable. With improvements such as are suggested a much more rigid test could be made.

Queen's University, Kingston, Canada, December 17 .

The Message of Science.

Mr. W. Robertson's letter in Nature of January 5 is very timely. May I invite him and those his letter has interested or impressed to put into practice, in Middlesbrough or any other town, the "most practical suggestion of immediate value" he describes, with one important addition. Some of us have recently been striving to get his suggestion, thus widened and clinched, made operative in other British centres of population on behalf of the British Science Guild, the objective of which comprehends the exact duty Mr. Robertson acclaims and the methods of which in a new campaign have been dictated by a lively sense of provincial and metropolitan needs in the harnessing of science to important public tasks.

Our methods begin just where those of so many other people leave off. We ask local scientific societies and organisations and all who desire to make their special scientific equipment of use to their times to establish touch at once with the important local organisations and groups in which business men, administrators, and the occupational classes gather, and with them to consider "the progressive connecting of science with individual and corporate conduct," not in general. nor on another continent, but in their own towns (where facts can be known and methods tested), and in any of the problems on which much public time and public money have inevitably to be spent. That suffices for a beginning.

Many are feeling to-day that science should become, and can become, the "chief formative factor of modern 